\title{
Effectiveness of Wellbeing Intervention for Chronic Kidney Disease (WICKD): results of a randomised controlled trial
}

Kylie M. Dingwall ${ }^{*}$, Michelle Sweet ${ }^{1}$, Alan Cass², Jaquelyne T. Hughes ${ }^{2,3,4}$, David Kavanagh ${ }^{5}$, Kirsten Howard ${ }^{6}$, Federica Barzi ${ }^{2}$, Sarah Brown ${ }^{7}$, Cherian Sajiv ${ }^{8}$, Sandawana W. Majoni ${ }^{2,4,9}$ and Tricia Nagel ${ }^{2}$

\begin{abstract}
Background: End stage kidney disease (ESKD) is associated with many losses, subsequently impacting mental wellbeing. Few studies have investigated the efficacy of psychosocial interventions for people with ESKD and none exist for Indigenous people, a population in which the ESKD burden is especially high.

Methods: This three-arm, waitlist, single-blind randomised controlled trial examined efficacy of the Stay Strong App in improving psychological distress (Kessler distress scale; K10), depressive symptoms (adapted Patient Health Questionnaire; PHQ-9), quality of life (EuroQoL; EQ. 5D) and dialysis adherence among Indigenous Australians undergoing haemodialysis in central and northern Australia (Alice Springs and Darwin), with follow up over two 3month periods. Effects of immediate AlMhi Stay Strong App treatment were compared with those from a contact control app (The Hep B Story) and treatment as usual (TAU). Control conditions received the Stay Strong intervention after 3 months.

Results: Primary analyses of the full sample $(N=156)$ showed statistically significant decreases in $\mathrm{K} 10$ and PHQ-9 scores at 3 months for the Hep B Story but not for the Stay Strong app or TAU. Restricting the sample to those with moderate to severe symptoms of distress or depression (K10 $>=25$ or PHQ-9 $>=10$ ) showed significant decreases in K10 and PHQ-9 scores for both Stay Strong and Hep B Story. No significant differences were observed for the EQ-5D or dialysis attendance.

Conclusions: Findings suggest that talking to people about their wellbeing and providing information relevant to kidney health using culturally adapted, locally relevant apps improve the wellbeing of people on dialysis. Further research is required to replicate these findings and identify active intervention components.
\end{abstract}

Trial registration: ACTRN12617000249358; 17/02/2017.

Keywords: Renal, E-mental health, Indigenous, Wellbeing, Kidney disease

\footnotetext{
* Correspondence: kylie.dingwall@menzies.edu.au

${ }^{1}$ Menzies School of Health Research, Charles Darwin University, Alice Springs, NT 0870, Australia

Full list of author information is available at the end of the article
}

(C) The Author(s). 2021 Open Access This article is licensed under a Creative Commons Attribution 4.0 International License, which permits use, sharing, adaptation, distribution and reproduction in any medium or format, as long as you give appropriate credit to the original author(s) and the source, provide a link to the Creative Commons licence, and indicate if changes were made. The images or other third party material in this article are included in the article's Creative Commons licence, unless indicated otherwise in a credit line to the material. If material is not included in the article's Creative Commons licence and your intended use is not permitted by statutory regulation or exceeds the permitted use, you will need to obtain permission directly from the copyright holder. To view a copy of this licence, visit http://creativecommons.org/licenses/by/4.0/ The Creative Commons Public Domain Dedication waiver (http://creativecommons.org/publicdomain/zero/1.0/) applies to the data made available in this article, unless otherwise stated in a credit line to the data. 


\section{Background}

End-stage kidney disease (ESKD) is the most severe form of chronic kidney disease (CKD) and requires a transplant or dialysis for survival. In Australia, rates of ESKD are higher among the elderly, Indigenous Australians and people living in remote and socioeconomically disadvantaged areas [1-3]. Australia's Northern Territory is a large geographical area consisting of a relatively small population of non-Indigenous people living mostly in urban centres along with smaller Indigenous groups living mostly in remote settings distant from centralised services [4]. The majority of Indigenous ESKD patients receive ongoing haemodialysis treatment in a hospital satellite unit setting. For Indigenous patients in the NT, access to haemodialysis usually requires relocation to urban centres adding to the already high emotional burden associated with ESKD.

Indigenous patients are known to demonstrate extraordinary 'tenacity and 'resilience' in the face of ESKD and its impacts [5]. Nevertheless, depression can be common in people undergoing dialysis (25\% show depressive symptoms when assessed by clinical interview, $40 \%$ when assessed by self-report measures) [6] with depressive symptoms considered a risk factor for poor outcome [7].

Psychosocial interventions for ESKD may prevent or minimise impact of debilitating mental disorders and wellbeing concerns, however well designed intervention studies are lacking for both mainstream and Indigenous CKD populations [7, 8]. Furthermore, there is a significant lack of rigorous effectiveness trials for mental health interventions in an Indigenous context generally [9].

Recent technological advancements and government initiatives have rendered digital mental health $(\mathrm{dMH})$ interventions more popular due to their accessibility and increasing evidence [10-12]. Culturally responsive, strengths-based, early-intervention mental health treatment programs are considered most appropriate to influence the high rates of psychological distress and suicide experienced by Indigenous Australians [13]. The Aboriginal and Islander Mental Health Initiative (AIMhi) Stay Strong care plan is a culturallyadapted, well-researched, effective engagement and low-intensity treatment strategy for improving Indigenous SEWB [14-17]. It adopts an empowering, person-centred, holistic and strengths based approach which acknowledges and promotes Indigenous cultural and family values and client self-management [18] and has recently been translated into a digital (tablet) format (the AIMhi Stay Strong App) and adapted for the Indigenous ESKD setting [19, 20].

This study aimed to determine whether the AIMhi Stay Strong App improves mental health and wellbeing for Indigenous people receiving haemodialysis, relative to delayed-treatment control groups at 3 months, and whether benefits are maintained at 6 months postrecruitment.

\section{Methods \\ Hypotheses}

We hypothesised that the AIMhi Stay Strong App would be superior to both a contact control (another app - Hep B Story) [21] and usual care, in reducing psychological distress and depressive symptoms, and improving quality of life, and dialysis adherence at 3 months. We also expected that delivering the Stay Strong app to control groups after the 3-month assessment would result in those groups showing improvements in these outcomes between 3 and 6 months.

\section{Study design and participants}

The study methods have been detailed previously [17] and are summarised below.

This was a three-arm, waitlist, single-blind randomised controlled trial with 2:2:1 allocation ratio testing the efficacy of the Stay Strong App intervention in improving wellbeing among Indigenous clients undergoing haemodialysis for ESKD in Alice Springs and Darwin. Assessments occurred at Baseline, 3 and 6 months (see Fig. 1 for CONSORT diagram). The three treatment conditions were: 1) Immediate treatment with the Stay Strong App ("ISS"), 2) Contact control/Delayed Stay Strong treatment (i.e. patients are engaged with the researcher for a similar time using the Hep B Story app then received the Stay Strong App at 3 months; "HepB/DSS"), and 3) Treatment as usual/Delayed Stay Strong treatment after 3 months ("TAU/DSS"; see Fig. 1).

Inclusion criteria were Aboriginal and Torres Strait Islander and aged $\geq 18$ years, receiving maintenance haemodialysis in Alice Springs or Darwin for more than 6 months. Exclusion Criteria were aged < 18 years, guardianship order in place, or inability to provide informed consent (e.g. because of cognitive or visual impairment). No major changes to the study protocol occurred after trial commencement.

\section{Consent, ethics and culturally appropriate approach}

This study was performed in accordance with the Declaration of Helsinki. Approvals were granted by the Central Australian Human Research Ethics Committee (CAHREC No: HREC-16-406) and the Human Research Ethics Committee (HREC) for the NT Department of Health and Menzies School of Health Research (HREC16-2599), including an Aboriginal subcommittee. Fully informed oral consent was obtained from all participants using pictorial information sheets and flipcharts in plain English with Aboriginal language versions available. 


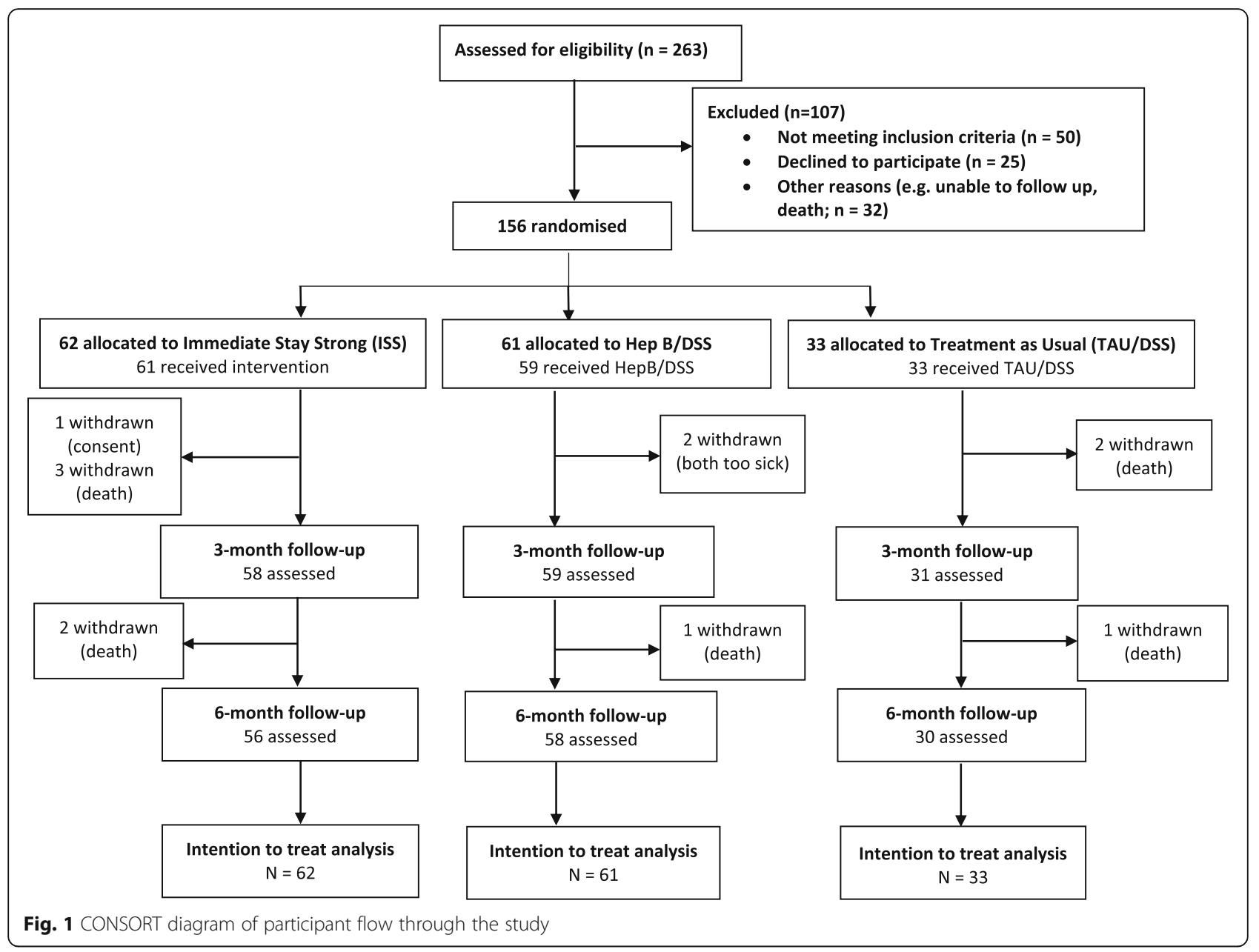

Demographic information and outcome measures were collected using a tablet device including pictorial prompts and Aboriginal language recordings (choice of 11 NT languages). Interpreters were utilised where necessary.

\section{Interventions}

In addition to their allocated treatment, all participants received usual care from their renal service. Usual care was carried out according to the norms prevailing in the renal service, informed by the needs of the client. Interventions are described in detail elsewhere and outlined below [17].

\section{Immediate AIMhi stay strong app treatment (ISS)}

Baseline Participants randomised to ISS completed a 20-min interview using the AIMhi Stay Strong App at baseline, with a second $\sim 20$-min session using the App within 2-4 weeks. Session 1 explored family, strengths, worries and goal setting. Session 2 reviewed information entered previously, refined the goals and addressed any barriers to goal attainment, setting new goals as appropriate. Participants received a text message or phone call 1 week following the initial treatment reminding them of their goals and steps for making changes.

Three Months Participants received a further two sessions using the AIMhi Stay Strong app following the 3month follow-up assessment. The two 20-min sessions occurred 2-4 weeks apart following a similar format to the earlier sessions. A text message or phone call was sent 1 week following the initial treatment to remind participants of their goals and steps for making change and the time for the next session.

\section{Hep B story contact control/delayed stay strong treatment (HepB/DSS)}

Baseline Participants randomised to HepB/DSS received 20 min of contact with the researcher using a culturally appropriate health app (i.e. The Hep B Story) at baseline, with a further 20-min session using the same app after 2-4 weeks. This ensured that each group received the 
same contact time and controlled for use of an app to structure the control session, as well as assisting with participant blinding. In Session 1 the participant interacted with the Hep B Story app with support from the researcher, focusing on app navigation and content. A 'goal' to talk to someone else in their family about the app content before the next session was set. Participants received a pictorial summary (utilising similar colours and images to the intervention summary). Session 2 reviewed the information discussed in Session 1. Participants received a text message or phone call reminding them of their goal (to talk with someone about the information) in the intervening weeks.

Three months Participants received a 20-min interview using the AIMhi Stay Strong App (on a tablet device) following their 3-month assessment, with one further 20-min session using the App within 2-4 weeks, following the format of the sessions received by the ISS group at baseline.

\section{Treatment as usual/delayed stay strong treatment (TAU/ DSS)}

Baseline Participants who are randomised to TAU/DSS only received the questionnaires and no other researcher intervention at baseline.

Three months After the 3-month assessment, participants received the AIMhi Stay Strong app intervention, using the same procedures as the HepB/DSS group.

\section{Fidelity of the intervention}

The interventions were delivered by trained researchers with reference to the AIMhi Stay Strong Planning Brief Treatment Manual [22]. Reviews of App data and ongoing booster sessions were used by the research team to provide regular feedback to redirect and adjust their mode of delivery as needed [17]. We defined an acceptable level of fidelity as two sessions of treatment delivered, one of which was at least $15 \mathrm{~min}$. This applied to both the contact control (Hep B app) and intervention (Stay Strong app).

\section{Outcomes}

All participants were assessed at the beginning of the study (T0), after 3 months (T3) and again after 6 months (T6) with the following instruments: (1) the Kessler Distress Scale $(K 10,2)$ the adapted Patient Health Questionnaire (PHQ-9); and the EuroQol (EQ-5D) 5 level. The $\mathrm{K} 10$ is a 10-item measure of psychological distress that is sensitive to symptoms of both anxiety and depression [23]. Responses are on a 5-point Likert scale. The PHQ-9 is a 9-item measure of depressive symptoms, scored on a 4-point Likert scale and has been adapted and tested for Australian Aboriginal people [24]. EQ-5D is a self-report measure of quality of life in 5 domains (mobility, self-care, usual activities, pain and discomfort, anxiety and depression). Respondents rate their health today on 5 levels of severity. It also includes a visual analogue scale which is used as a quantitative measure of overall health status. These assessments and their justification are described in detail elsewhere [17].

\section{Sample size and power}

The sample size calculation aimed to detect a minimum difference of 5 points on the K10 (with 90\% power and an alpha of .05) leading to a sample size of 62, 62 and 32 for the ISS, HepB/DSS and TAU/DSS groups respectively. Based on previous data we considered a difference between the group mean scores of 5 to be clinically significant. This calculation allowed for $10 \%$ attrition.

\section{Randomisation}

Participants were randomised using a block sequential random number sequence (block sizes of $2 \& 3$ ) and an envelope system of randomisation, stratified by site, level of psychological distress (high or low i.e. $<$ or $>25$ ) and access to respite dialysis in home community as a person's ability to return home to family (for visits) may influence wellbeing. An independent statistician created the allocation schedule with a computerized random number generator and investigators were blind to this schedule. Following baseline assessment, research assistants selected the next envelope in the sequence based on the participants site, level of distress and access to dialysis at home. Participants were allocated to ISS, HepB/DSS or TAU/DSS at a ratio of 2:2:1. The allocation was concealed to participants.

\section{Blinding}

Participants and outcome assessors were blinded to treatment condition. Research assistants who delivered the intervention were restricted from conducting follow up assessments with those participants to maintain blinding. The contact control involved an intervention of similar length of time and utilised an app presented on a tablet device to minimise client awareness of their treatment group assignment.

\section{Statistical analyses}

The primary analyses were conducted according to the intention-to-treat principle.

Descriptive statistics of baseline characteristics are provided by randomisation group. Continuous variables are summarised with mean and standard deviation (SD) when appropriate, or median and interquartile range 
(IQR) when not normally distributed, categorical variables were summarized with frequency and percentage.

Linear mixed models were used to estimate, for each of the K10, the EQ-5D and PHQ-9 score, the change between: (i) baseline assessment and 3-month assessment (ii) baseline and 6-month assessment (iii) 3-month and 6-month assessment. The changes described above were also compared between groups (ISS vs HepB/DSS; ISS vs TAU/DSS; HepB/DSS vs TAU/DSS) with mixed models that included a categorical variable for the three intervention arms, for each time point and an interaction term between arm and time. The use of mixed models has the advantage of allowing the modelling of the correlation between repeats of outcome recorded within the same participant and within site. Sensitivity analyses including baseline scores were included in the models when descriptive analyses showed important between intervention arm differences for baseline scores. The above described analyses were carried out for subgroups defined by baseline score severity (below or above 25 for K10 and below and above 10 for the PHQ-9), by region (Top End and Central Australia), access to home community dialysis or not.

Zero inflated Poisson models were used to calculate and compare between allocation arms, mean and 95\% confidence intervals of numbers of missing dialysis sessions during the baseline to 3-month period, and the 46-month period.

\section{Results}

\section{Participants}

Sixty-two participants were allocated to receive ISS, 61 to HepB/DSS, and 33 to TAU/DSS. Data collection occurred between February 2017 and March 2019. Participant flow is described in Fig. 1. Baseline descriptive statistics are presented in Table 1. Nine participants died during the study, for reasons unrelated to the trial and consistent with the high mortality experienced generally amongst the dialysis population, and two were withdrawn due to being too ill to participate (see Fig. 1). Follow up at 3 months as achieved for $93.4 \%$ of the ISS group, $96.7 \%$ of the HepB/DSS group and $93.9 \%$ of the TAU/DSS group. At 6 months, follow up was achieved for $90.3,95.1$ and $90.9 \%$ respectively. Overall attrition was $7.7 \%$. The trial ended when the proposed sample size was achieved.

\section{Fidelity}

Table 2 reports number of missed sessions and average minutes for each session by treatment group. For the ISS arm, the number who received the intervention with an acceptable level of fidelity ( 2 sessions, one of which was greater than or equal to $15 \mathrm{~min}$ ) was $48 / 61$ (79\%) the early treatment period and $37 / 56(66 \%)$ at the delayed treatment period. For the Hep B/DSS group, the number who received the intervention with an acceptable level of fidelity (described above) was 41/59 (70\%) for the early (i.e. Hep B) treatment period and 45/58 (78\%) for the delayed (i.e. Stay Strong) treatment period. For TAU/DSS, 18/30 (60\%) received Stay Strong with an acceptable level of fidelity at the delayed treatment period.

\section{Changes in $\mathrm{K} 10$ over time and between arms}

Participants in the HepB/DSS group showed a statistically significant decrease ( 2.5 points) in $\mathrm{K} 10$ score from baseline to 3 -months (95\% CI: $0.5-4.6 ; p=0.02$ ) and more so from baseline to 6 months with a decline of 3.5 points (95\% CI: $1.5-5.7 ; p=0.001$ ) however these changes were not clinically significant (i.e. $>=5$ points). Significant reductions in K10 scores in the ISS and TAU/DSS arms were not evident. There was no substantial difference in scores between groups over time, however, when compared to ISS, HepB/DSS had a

Table 1 Summary of baseline characteristics by intervention group

\begin{tabular}{|c|c|c|c|c|c|}
\hline & & $\begin{array}{l}\text { ISS } \\
N=62\end{array}$ & $\begin{array}{l}\text { HepB/DSS } \\
N=61\end{array}$ & $\begin{array}{l}\text { TAU/DSS } \\
N=33\end{array}$ & $\begin{array}{l}\text { Total } \\
N=156\end{array}$ \\
\hline \multirow[t]{2}{*}{ Gender: } & Male & $16(25.8)$ & $19(31.2)$ & $9(27.3)$ & $44(28.2)$ \\
\hline & Female & $46(74.2)$ & $42(68.9)$ & $24(72.7)$ & $112(71.8)$ \\
\hline Age at randomisation: & (years) & $55(10.6)$ & $53.9(8.7)$ & $57.0(8.2)$ & $55(9.4)$ \\
\hline Years since initiation of dialysis & Median [IQR] & $3.4[2.1-8.5]$ & $2.8[1.6-5.2]$ & $3.6[2.4-5.7]$ & $3.1[2.0-5.7]$ \\
\hline English as 1st language & N (\%) & $10(16 \%)$ & $11(18 \%)$ & $7(21 \%)$ & $28(18 \%)$ \\
\hline Access to dialysis at home community & N (\%) & $37(60 \%)$ & $38(62 \%)$ & $22(67 \%)$ & $97(62 \%)$ \\
\hline Top End & N (\%) & $32(52 \%)$ & $33(54 \%)$ & $13(39 \%)$ & $78(50 \%)$ \\
\hline Central Australia & N (\%) & $30(48 \%)$ & $28(46 \%)$ & $20(61 \%)$ & $78(50 \%)$ \\
\hline $\mathrm{K}-10$ & Mean (SD) & $21.4(7.4)$ & $23.6(8.6)$ & $22.3(8.8)$ & $22.4(8.2)$ \\
\hline PHQ-9 & Mean (SD) & $8.4(5.2)$ & $9.1(5.7)$ & $7.9(4.4)$ & $8.6(5.2)$ \\
\hline EQ-5D & Mean (SD) & $74.5(21.7)$ & 70.1 (18.2) & $77.9(17.2)$ & 73.5 (19.6) \\
\hline
\end{tabular}


Table 2 Fidelity data by intervention group

\begin{tabular}{|c|c|c|c|c|c|c|}
\hline & \multicolumn{2}{|c|}{ Immediate Stay Strong (ISS) } & \multicolumn{2}{|l|}{ HepB/DSS } & \multicolumn{2}{|l|}{ TAU/DSS } \\
\hline & Number missed & Average minutes & Number missed & Average minutes & Number missed & Average minutes \\
\hline \multicolumn{7}{|c|}{ Immediate Treatment } \\
\hline session 1 & $1 / 62$ & 24.09 & $3 / 61$ & 17.55 & - & - \\
\hline session 2 & $7 / 62$ & 19.04 & $13 / 61$ & 15.15 & - & - \\
\hline \multicolumn{7}{|c|}{ Delayed Treatment - Stay Strong } \\
\hline session 1 & $5 / 62$ & 22.34 & $2 / 61$ & 24.86 & $5 / 33$ & 24.57 \\
\hline session 2 & $14 / 62$ & 16.30 & $9 / 61$ & 14.12 & $7 / 33$ & 15.31 \\
\hline
\end{tabular}

marginally greater decrease from baseline to 3-months [3.2 (0.3-6.1); $p=0.03$ ]. (Fig. 2; Table 3, 4).

The subgroup analyses by baseline severity indicated a statistically and clinically significant effect of the ISS intervention at 6 months for those with a baseline K10 greater than or equal to 25 . Similar results to both the ISS results and the primary analysis were found for the HepB/DSS group, with changes also attaining clinical significance (see Tables 5 \& 6). While there was no significant difference in mean changes between groups, there was a trend for ISS to show a marginally greater decrease in K10 scores than
TAU/DSS from 3 to 6 months $[6.0(0.1-12.0) p=$ 0.05] (see Fig. 3).

No significant decreases in K10 scores were observed in the subgroup analyses for any treatment arm when baseline K10 was less than 25 .

There were no substantive changes after adjusting by baseline K10 so unadjusted results are reported.

\section{Changes in PHQ-9 over time and between arms}

PHQ-9 changes were similar to those of K10. Primary analyses indicated, participants in the HepB/DSS group showed a statistically significant (but not

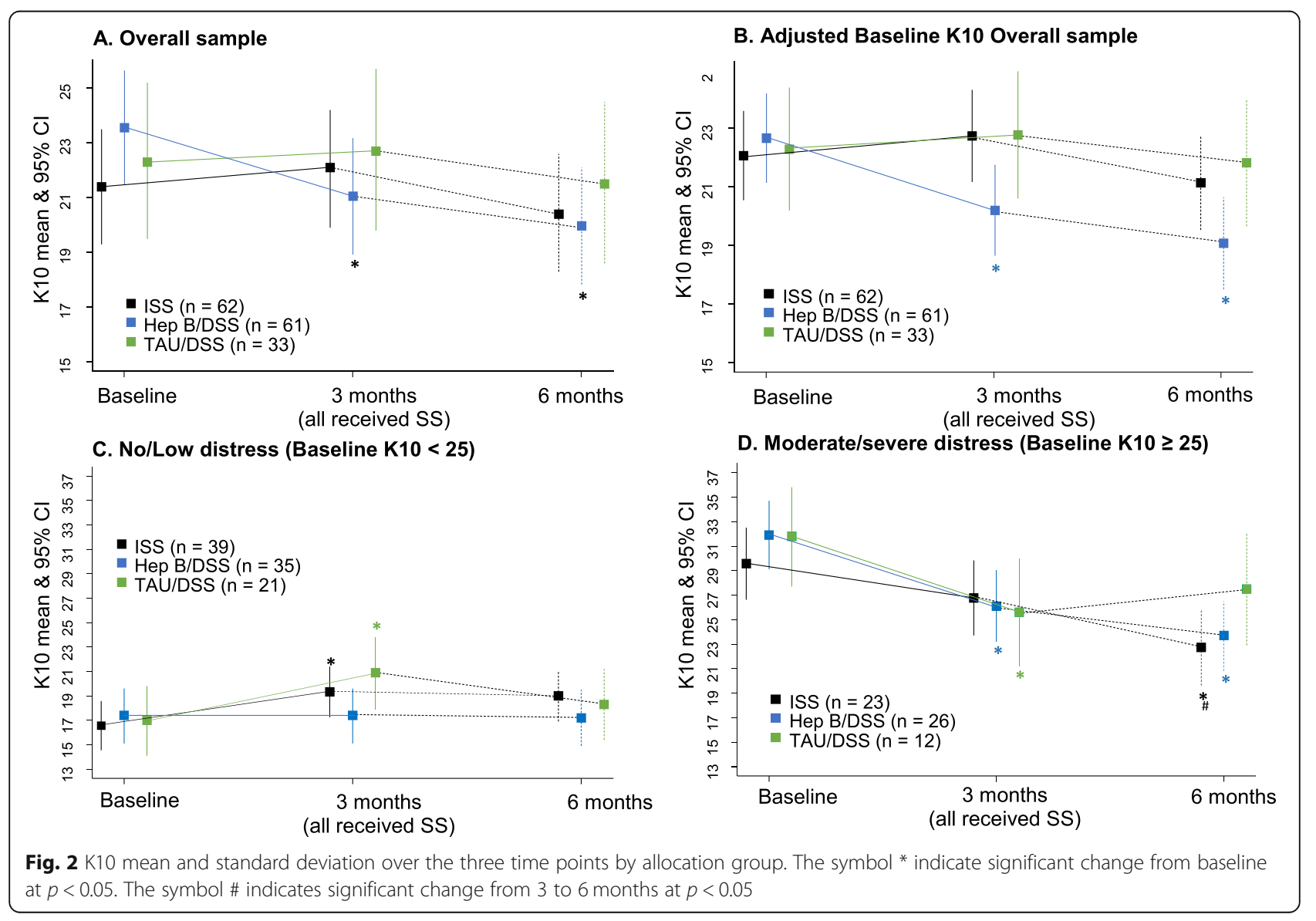


Table 3 Mean (and 95\%Cl) K10, PHQ-9, and EQ-5D scores at Baseline, 3-months and 6-months for full sample

\begin{tabular}{|c|c|c|c|c|c|c|c|}
\hline \multirow[t]{2}{*}{ Measure } & \multirow[t]{2}{*}{ Timepoint } & \multicolumn{2}{|l|}{ ISS } & \multicolumn{2}{|c|}{ HepB/DSS } & \multicolumn{2}{|c|}{ TAU/DSS } \\
\hline & & $n$ & Mean $(95 \% \mathrm{Cl})$ & $n$ & Mean $(95 \% \mathrm{Cl})$ & $n$ & Mean $(95 \% \mathrm{Cl})$ \\
\hline \multirow[t]{3}{*}{ K10 } & Baseline & 62 & $21.4(19.3-23.5)$ & 61 & $23.6(21.5-25.7)$ & 33 & $22.3(19.5-25.2)$ \\
\hline & 3-months & 58 & $22.1(19.9-24.2)$ & 59 & $21.1(18.9-23.2)$ & 30 & $22.7(19.8-25.7)$ \\
\hline & 6-months & 56 & $20.4(18.3-22.6)$ & 57 & $20.0(17.8-22.1)$ & 30 & $21.5(18.6-24.5)$ \\
\hline \multirow[t]{3}{*}{ PHQ-9 } & Baseline & 62 & $8.4(7.1-9.7)$ & 61 & $9.1(7.7-10.4)$ & 33 & $7.9(6.1-9.7)$ \\
\hline & 3-months & 58 & $8.2(6.8-9.6)$ & 59 & $7.5(6.1-8.9)$ & 30 & $8.8(6.9-10.6)$ \\
\hline & 6-months & 56 & $7.7(6.3-9.0)$ & 57 & $7.6(6.3-9.0)$ & 30 & $8.2(6.3-10.1)$ \\
\hline \multirow[t]{3}{*}{ EQ-5D } & Baseline & 62 & $0.78(0.72-0.84)$ & 31 & $0.79(0.72-0.85)$ & 33 & $0.83(0.74-0.91)$ \\
\hline & 3 months & 58 & $0.77(0.70-0.83)$ & 59 & $0.84(0.78-0.90)$ & 30 & $0.79(0.71-0.88)$ \\
\hline & 6 months & 56 & $0.82(0.75-0.88)$ & 57 & $0.83(0.76-0.89)$ & 30 & $0.82(0.73-0.91)$ \\
\hline
\end{tabular}

clinically significant) decrease (1.6 points) in the total PHQ-9 score from baseline to 3 months (95\% CI: $0.3-2.9 ; p=0.02$ which remained at 6 months $(p=$ 0.03). Significant reductions in PHQ-9 scores for the ISS and TAU/DSS arms were not evident. There was no substantial difference in PHQ-9 scores between groups over time, however, when compared to TAU/ DSS, HepB/DSS had a marginally greater decrease from baseline to 3 months [2.4 (0.2-4.7); $p=0.03]$. There was no adjustment made for baseline PHQ-9 scores given the similar scores between groups at baseline.

The subgroup analyses by baseline severity indicated an effect of the ISS for those with a baseline PHQ-9 greater than or equal to 10 . For this subgroup, there was a statistically significant decrease (4.08 points) in PHQ-9 score from baseline to 3 months which remained at 6 months. While reductions were less than the 5 points required to reach clinical significance, the changes approached clinical significance (4.08 points at 3 months and 4.68 points at 6 months). Similar results to this and the overall were found for the HepB/DSS group with changes failing to reach clinical significance (3.55 and 3.18 at 3 and 6 months respectively). Both ISS and HepB/DSS showed significantly larger decreases in PHQ-9 scores from baseline to 3 months and baseline to 6 months compared to TAU/DSS.

No significant decreases in PHQ-9 scores were observed in the subgroup analyses for any treatment arm when PHQ-9 was less than 10.

\section{Changes in EQ. 5D over time and between arms}

There were no significant changes over time nor any significant differences between the groups for the EQ-5D.

\section{Dialysis attendance}

There was no difference between groups in number of missed dialysis sessions at either 3 months or 6 months see Table 7 .

\section{Discussion}

The HepB/DSS group showed decreased levels of distress and depressive symptoms at 3 months across the whole sample, with changes maintained at 6 months (after also receiving the Stay Strong app) although these changes did not attain clinical significance. When restricted to moderate/severely distressed or depressed participants (K10 > $=25$ or PHQ-9 $>=10)$, participants in either the ISS or HepB/DSS groups demonstrated clinically significant changes in distress symptoms evident immediately for the HepB/DSS group (i.e. at 3 months) and

Table 4 Mean difference (and 95\% Cl) from Baseline to 3 months (TO-T3), Baseline to 6 months (T6-T0) and 3 months to 6 months (T6-T3) within allocated group and between groups for full sample

\begin{tabular}{llllllll}
\hline Measure & Timepoint & ISS & HepB/DSS & TAU/DSS & ISS vs HepB/DSS & ISS vs TAU/DSS & HepB/DSS vs TAU/DSS \\
\hline K10 & T3-T0 & $0.7(-1.4,2.8)$ & $-2.5(-4.6,-0.5)^{*}$ & $0.4(-2.4,3.3)$ & $3.2(0.3,6.1)^{*}$ & $0.3(-3.2,3.8)$ & $-2.9(-6.4,0.6)$ \\
& T6-T0 & $-1.0(-3.0,1.1)$ & $-3.6(-5.7,-1.5)^{* *}$ & $-0.8(-3.6,2.1)$ & $2.6(-0.3,5.6)$ & $-0.2(-3.7,3.4)$ & $-2.8(-3.3,0.7)$ \\
& T6-T3 & $-1.6(-3.8,0.5)$ & $-1.1(-3.2,1.0)$ & $-1.2(-4.1,1.7)$ & $-0.6(-3.5,2.4)$ & $-0.4(-4.0,3.1)$ & $0.1(-3.5,3.7)$ \\
PHQ-9 & T3-T0 & $-0.02(-3.2,0.4)$ & $-1.6(-2.9,-0.3)^{*}$ & $0.8(-1.0,2.7)$ & $1.4(-0.4,3.2)$ & $-1.0(-3.3,1.2)$ & $-2.4(-4.7,-0.2)$ \\
& T6-T0 & $-0.7(-2.1,0.6)$ & $-1.4(-2.8,-0.1)^{*}$ & $0.3(-1.6,2.1)$ & $0.7(-1.2,2.6)$ & $-1.0(-3.2,1.3)$ & $-1.7(-3.9,0.5)$ \\
& T6-T3 & $-0.5(-1.9,0.8)$ & $0.1(-1.2,1.5)$ & $-0.6(-2.4,1.2)$ & $-0.7(-2.6,1.2)$ & $0.1(-2.2,2.3)$ & $0.7(-1.5,3.0)$ \\
\hline
\end{tabular}


Table 5 Mean (and 95\%Cl) K10, and PHQ-9 scores at Baseline and 3-months and 6-months for sub-analyses by symptom severity

\begin{tabular}{|c|c|c|c|c|c|c|c|}
\hline \multirow[t]{2}{*}{ Measure } & \multirow[t]{2}{*}{ Timepoint } & \multicolumn{2}{|c|}{ ISS } & \multicolumn{2}{|c|}{ HepB/DSS } & \multicolumn{2}{|c|}{ TAU/DSS } \\
\hline & & $n$ & Mean \& 95\% Cl & $n$ & Mean \& 95\% Cl & $\mathrm{n}$ & Mean \& 95\% Cl \\
\hline \multirow[t]{3}{*}{$\mathrm{K} 10>=25$} & Baseline & 23 & $29.6(26.6-32.5)$ & 26 & $31.9(29.1-34.7)$ & 12 & $31.8(27.7-35.8)$ \\
\hline & 3-months & 21 & $26.8(23.7-29.8)$ & 24 & 26.1 (23.7-29.0) & 10 & $25.6(21.2-30.0)$ \\
\hline & 6-months & 20 & 22.7 (19.6-25.9) & 24 & 23.7 (20.8-26.5) & 9 & $27.5(22.9-32.1)$ \\
\hline \multirow[t]{3}{*}{$\mathrm{K} 10<25$} & Baseline & 39 & $16.6(14.5-18.6)$ & 35 & $17.3(15.1-19.6)$ & 21 & $17.0(14.1-19.9)$ \\
\hline & 3-months & 37 & $19.3(17.3-21.4)$ & 35 & $17.3(15.1-19.6)$ & 20 & 20.9 (17.9-23.8) \\
\hline & 6-months & 36 & $19.0(16.9-21.1)$ & 33 & $17.2(14.9-19.5)$ & 21 & $18.3(15.4-21.2)$ \\
\hline \multirow[t]{3}{*}{ PHQ-9 > =10 } & Baseline & 22 & $14.1(12.1-16.1)$ & 28 & $14.1(12.4-15.9)$ & 13 & $12.3(9.6-15.1)$ \\
\hline & 3-months & 21 & $10.0(8.0-12.1)$ & 27 & $10.6(8.8-12.4)$ & 11 & 13.6 (10.8-16.5) \\
\hline & 6-months & 20 & $9.4(7.3-11.5)$ & 27 & $11.0(9.1-12.8)$ & 10 & $13.7(10.8-16.7)$ \\
\hline \multirow[t]{3}{*}{ PHQ-9 $<10$} & Baseline & 40 & $5.3(4.1-6.4)$ & 33 & $4.8(3.5-6.0)$ & 20 & $4.9(3.3-6.5)$ \\
\hline & 3-months & 37 & $7.3(6.1-8.5)$ & 32 & $4.8(3.6-6.1)$ & 19 & $5.7(4.1-7.3)$ \\
\hline & 6-months & 36 & $6.8(5.6-8.0)$ & 30 & $4.7(3.4-6.0)$ & 20 & $5.0(3.4-6.5)$ \\
\hline
\end{tabular}

at 6 months for both. Similar results were shown for depressive symptoms with significant changes at both 3 months and 6 months (which only approached clinical significance for the ISS group). Our findings of significant improvements in participants with more severe symptoms accord with previous studies examining effectiveness of apps for reducing depression. These have shown variation in outcomes based on symptom severity [25]. Future studies might therefore be more targeted by screening for symptom severity, as a floor effect will likely occur in participants with few symptoms: further improvement is both less likely and more difficult to detect in those who are already well.

As expected, baseline symptoms of depression and distress were relatively common in this group, with $45 \%$ (70/156) of participants scoring in the moderate/severe range on the PHQ-9 (i.e. $>=10)$ and 39\% (61/156) scoring in the moderate/severe range on the K10 (i.e. > = 25). Rates of depressive symptoms were consistent with meta analyses previously reporting rates of $39 \%$ of dialysis patients experiencing depressive symptoms when assessed with an assessment tool [6]. However, previous

Table 6 Mean difference (and 95\% Cl) from Baseline to 3 months (T0-T3), Baseline to 6 months (T6-T0) and 3 months to 6 months (T6-T3) within allocated group and between groups by symptom severity

\begin{tabular}{|c|c|c|c|c|c|c|c|}
\hline Measure & Timepoint & ISS & HepB/DSS & TAU/DSS & $\begin{array}{l}\text { ISS vs HepB/ } \\
\text { DSS }\end{array}$ & ISS vs TAU/DSS & $\begin{array}{l}\text { HepB/DSS vs } \\
\text { TAU/DSS }\end{array}$ \\
\hline \multirow[t]{3}{*}{$\mathrm{K} 10>=25$} & Т3-Т0 & $-2.8(-6.1,0.5)$ & $-5.8(-8.9,-2.7)^{* *}$ & $\begin{array}{l}-6.2(-10.9 \\
-1.5)^{*}\end{array}$ & $3.0(-1.5,7.5)$ & $3.4(-2.4,9.2)$ & $0.4(-5.3,6.0)$ \\
\hline & T6-T0 & $\begin{array}{l}-6.8(-10.2 \\
-3.5)^{* *}\end{array}$ & $\begin{array}{l}-8.2(-11.3,- \\
5.1)^{* *}\end{array}$ & $-4.2(-9.1,0.7)$ & $1.4(-3.2,5.9)$ & $-2.6(-8.5,3.3)$ & $-4.0(-9.8,1.8)$ \\
\hline & T6-T3 & $-4.0(-7.4,-0.7)^{*}$ & $-2.4(-5.5,0.7)$ & $2.0(-3.1,7.0)$ & $-1.6(-6.2,3.0)$ & $-6.0(-12.0,0.1) \sim$ & $-4.4(-10.3,1.5)$ \\
\hline \multirow[t]{3}{*}{$\mathrm{K} 10<25$} & Т3-Т0 & $2.8(0.5,5.1)^{*}$ & $0.0(-2.4,2.4)$ & $3.9(0.8,7.0)^{*}$ & $2.8(-0.5,6.1)$ & $-1.2(-5.0,2.7)$ & $-3.9(-7.8,0.01)$ \\
\hline & T6-T0 & $2.4(0.1,4.8)^{*}$ & $-0.1(-2.6,2.3)$ & $1.3(-1.7,4.4)$ & $2.6(-0.8,5.9)$ & $1.1(-2.7,4.9)$ & $-1.5(-5.4,2.4)$ \\
\hline & T6-T3 & $-0.3(-2.7,2.0)$ & $-0.1(-2.6,2.3)$ & $-2.6(-5.7,0.5)$ & $-0.2(-3.6,3.2)$ & $2.3(-1.6,6.2)$ & $2.5(-1.5,6.4)$ \\
\hline \multirow[t]{3}{*}{$\begin{array}{l}\text { PHQ-9 > = } \\
10\end{array}$} & T3-Т0 & $-4.1(-6.4,-1.8)^{* *}$ & $-3.5(-5.6,-1.5)^{*}$ & $1.3(-1.9,4.4)$ & $-0.5(-3.6,2.5)$ & $-5.4(-9.3,-1.5)^{*}$ & $\begin{array}{l}-4.8(-8.6,- \\
1.1)^{*}\end{array}$ \\
\hline & T6-T0 & $-4.7(-7.0,-2.4)^{* *}$ & $-3.2(-2.2,-1.2)^{*}$ & $1.4(-1.9,4.6)$ & $-1.5(-4.6,1.6)$ & $\begin{array}{l}-6.1(-10.1,- \\
2.1)^{*}\end{array}$ & $-4.6(-8.4,-0.7)^{*}$ \\
\hline & T6-T3 & $-0.6(-2.9,1.7)$ & $0.4(-1.7,2.4)$ & $0.1(-3.2,3.4)$ & $-1.0(-4.1,2.1)$ & $-0.7(-4.7,3.3)$ & $0.3(-3.6,4.1)$ \\
\hline \multirow[t]{3}{*}{ PHQ-9 $<10$} & T3-T0 & $2.0(0.6,3.4)^{*}$ & $0.0(-1.5,1.5)$ & $0.8(-1.4,2.7)$ & $2.0(0.0,4.1)$ & $1.2(-1.1,3.6)$ & $-0.8(-3.2,1.7)$ \\
\hline & T6-T0 & $1.5(0.1,2.9)^{*}$ & $-0.1(-1.6,1.5)$ & $0.0(-1.9,2.0)$ & $1.6(-0.5,3.7)$ & $1.5(-0.9,3.8)$ & $-0.1(-2.6,2.3)$ \\
\hline & T6-T3 & $-0.5(-1.9,0.9)$ & $-0.1(-1.6,1.4)$ & $-0.7(-2.7,1.2)$ & $-0.4(-2.5,1.7)$ & $0.2(-2.2,2.6)$ & $0.6(-1.8,3.1)$ \\
\hline
\end{tabular}



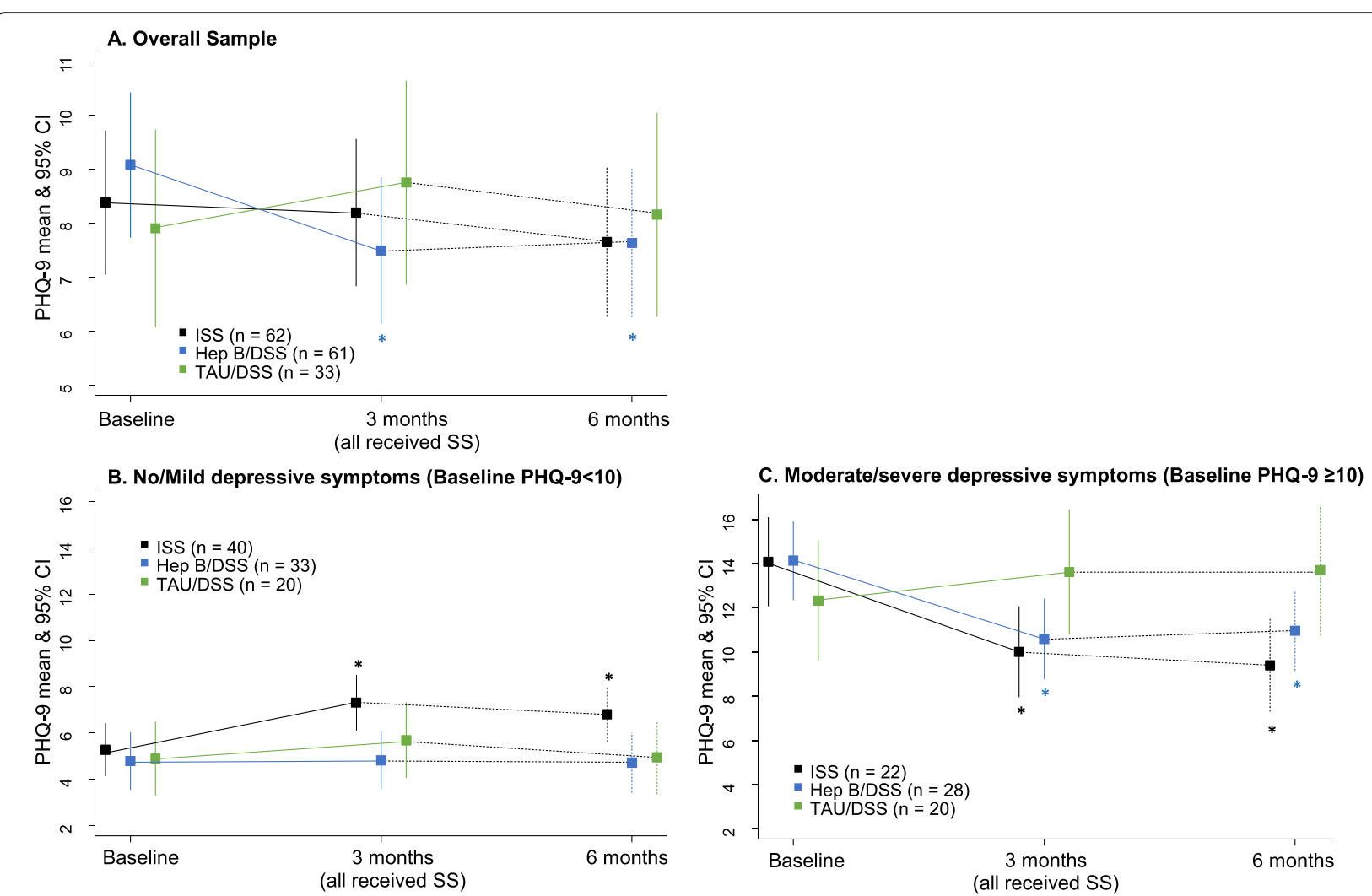

Fig. 3 PHQ-9 mean and standard deviation over the three time points by allocation group. The symbol * indicates significant change from baseline at $p<0.05$

research also suggests that cut off scores for assessment measures should be modified for those on dialysis given the overlap between somatic symptoms of depression and of dialysis itself [26].

Taken together, the results suggest that delivering culturally responsive app-based health assessments and interventions to Indigenous patients on dialysis can result in improvements in wellbeing. Given that depression is often underdiagnosed and undertreated in this group [26], this study suggests promising results can occur with relatively brief app-guided intervention. Brief, guided interventions such as those described here have potential to overcome some of the barriers to treatment described elsewhere, such as an already high medication burden, limiting willingness to add pharmacological treatments, or lack of motivation, resources or time for behavioural interventions [27]. Other reasons for low treatment rates include the lack of randomised controlled trial evidence demonstrating efficacy and safety of treatment regimens in CKD patients, confirming the importance of studies such as this one [26].

The Hep B app, which was chosen as a contact control, performed surprisingly well. It resulted in immediate change in distress and depressive symptoms (baseline to 3 months) which were maintained at 6 months after also receiving the Stay Strong App. On reflection, the Hep B Story was well aligned with participant identified needs and priorities, such as physical health and family. It was introduced as an intervention to promote physical health, with the goal at the end of the app being to share the information with family. It was also a less intense app to complete requiring a lower level of attention and concentration, and was available in one (top end) Indigenous language.

Table 7 Average number of missing dialysis sessions (\& 95\% confidence Intervals) over the study follow-up dialysis

\begin{tabular}{|c|c|c|c|c|c|c|c|c|c|c|}
\hline \multirow[b]{3}{*}{ ISS } & \multicolumn{4}{|c|}{$0-3$ months } & \multirow{3}{*}{$\begin{array}{l}P \text { value } \\
\text { comparing to ISS }\end{array}$} & \multicolumn{4}{|c|}{ 4-6 months } & \multirow{3}{*}{$\begin{array}{l}P \text { value } \\
\text { comparing to ISS }\end{array}$} \\
\hline & \multirow{2}{*}{$\begin{array}{l}\mathbf{n} \\
60\end{array}$} & \multirow{2}{*}{$\begin{array}{l}\text { mean } \\
4.3\end{array}$} & \multicolumn{2}{|c|}{$95 \% \mathrm{Cl}$} & & \multirow{2}{*}{$\begin{array}{l}\mathbf{n} \\
55\end{array}$} & \multirow{2}{*}{$\frac{\text { mean }}{3.6}$} & \multicolumn{2}{|c|}{$95 \% \mathrm{Cl}$} & \\
\hline & & & 3.5 & 5.1 & & & & 2.8 & 4.4 & \\
\hline HepB/DSS & 60 & 4.3 & 3.4 & 5.1 & 0.75 & 58 & 4.7 & 3.8 & 5.5 & 0.17 \\
\hline TAU/DSS & 30 & 2.9 & 1.9 & 4.0 & 0.47 & 32 & 2.4 & 1.5 & 3.4 & 0.31 \\
\hline
\end{tabular}


TAU/DSS appeared to show improvements in psychological distress (but not depressive symptoms) at 3 months for moderate/severely distressed participants. This change was not clinically significant and might be due to the small sample size in the sub analyses or related to spontaneous resolution of symptoms. The outcome assessments involved discussion of wellbeing and were delivered in local languages via an app. In consequence, it could be considered a digital mental health intervention. Assessment has previously been shown to have potential for a therapeutic effect, particularly when involving feedback [28]. Participants who scored highly on the K10 received follow up questions (per risk protocol) and were referred. Participants commented on the assessment app during the study stating, "It's good. No one has ever talked to me about this before".

The addition of a Stay Strong intervention at 3 months for those who had received the Hep B Story at baseline did not result in further improvement, but the gains made were maintained. Lack of improvement from 3 to 6 months may be due to significant improvements observed already in both HepB/DSS and TAU/DSS groups from baseline to 3 months, and thus no room for further significant improvement. It is unclear whether differences between the groups might have been observed at longer follow-up periods (e.g. 12 months), after participants had had a chance to practice the skills gained through receiving the Stay Strong intervention (and having these reinforced a second time for the intervention group). However, a 12-month follow up was considered difficult in this population given the high morbidity and mortality. Nine participants died during the study period, five of whom were in the Stay Strong intervention group.

Further limitations included the small sample size, particularly for sub-analyses, and the relatively short follow up period. Hence, findings should be interpreted with caution. Lack of a contact control group without the use of an app might also be considered a limitation.

No changes in EQ-5D scores were observed for this group and the utility of this measure in this setting is unclear. This measure was included as a quality of life measure to contribute to the cost-effectiveness analysis (publication in preparation) in order to calculate QALYs, however researcher experience of its use suggests its ability to tap into the quality of life domains valued by this population may be limited $[29,30]$. Similarly, no significant differences were identified between the groups on number of missed dialysis sessions, suggesting no impact of the interventions on treatment adherence. While literature suggests depression treatment can improve depressive symptoms in people with kidney disease, it is still unclear whether such therapy improves physical health outcomes [31].
More research is required to understand the specific active components of the interventions [32]. One potential active component is clinician guidance. Previous literature suggests that effect sizes of treatment apps can be influenced by clinician or other guidance, with guided interventions demonstrating a larger effect on outcomes than unguided interventions [33]. Both active conditions (Stay Strong app and Hep B app) were clinician-guided, which may have contributed to our results.

While the interventions in this study were developed specifically for Indigenous Australians, the findings have relevance for other first nations or minority populations internationally $[34,35]$. Other first nations groups share the heavy burden of ESKD and its associated challenges including separation from country and family, cultural isolation, uncertainty around accommodation and establishing life in an urban context, similarly impacting perceived quality of life [36]. Furthermore, divergent health beliefs between health care providers and minority groups are identified as challenges to delivery of effective health care with culturally appropriate communication strategies needed to address this issue [36]. This study showed that culturally adapted digital mental health interventions can be acceptable and effective. Meaningful user involvement in the development of mobile health technologies has been found to increase engagement and acceptability however, evidence for effectiveness remains limited [34]. The Framework for the Development and Evaluation of RCTs for Complex Interventions to Improve Health recognises there are specific difficulties in testing complex interventions and recognises that complex interventions may work best if they are tailored to local contexts [32]. Although acceptability and engagement are commonly assessed, and greater attention to early phase piloting and development work has been recommended, a focus on high quality RCTs such as this one is also needed to improve access to evidence-based, effective interventions to improve wellbeing and quality of life for first nations and other minority groups internationally.

\section{Conclusion}

This is the first RCT to examine efficacy of a dMH tool in an Indigenous CKD setting. Findings suggest that using apps for treatment can improve wellbeing for people on dialysis. Wellbeing apps like Stay Strong may work best for those who already have symptoms of distress or depression, and when delivered more than once. Simple, brief apps that focus on physical health through storytelling may also lead to important improvements in wellbeing. Further research is required to replicate these findings and identify active intervention components. 


\section{Acknowledgements}

Authors would like to thank all the staff and patients at NT Renal Services and Western Desert Nganampa Walytja Palyantjaku Tjutaku in Darwin and Alice Springs, Australia for their assistance with this study.

\section{Authors' contributions}

$\mathrm{KD}$ and $\mathrm{TN}$ were major contributors to the conception and design of the study, data interpretation and presentation and drafted the manuscript text and prepared Fig. 1. KD prepared Tables 2, 5, 6 and 7. MS was a major contributor to the conception and design of the study, conducted data collection and data analysis and assisted with writing and reviewing the manuscript. DK, AC, JH, KH, were major contributors to the conception and design of the study and assisted with writing and reviewing the manuscript. CS, SM, and SB contributed to the design of the study, and assisted with writing and reviewing the manuscript. FB conducted the statistical analysis and prepared Figs. 2 \& 3 and Tables 1, 3, and 4, and assisted with writing and reviewing the manuscript. All authors read and approved the final manuscript.

\section{Funding}

This project is supported by a National Health and Medical Research Council (NHMRC) project grant (GNT\# 1098311). JH was supported by NHMRC Fellowship (GNT\# 1092576). The funding source had no input into the design of the study or the preparation of this manuscript and the views expressed in this publication are those of the authors and do not reflect the views of NHMRC.

\section{Availability of data and materials}

The datasets generated and/or analysed during the current study are not publicly available due to participant confidentiality but may be available from the corresponding author on reasonable request.

\section{Declarations}

\section{Ethics approval and consent to participate}

This study was performed in accordance with the Declaration of Helsinki and approved by the Central Australian Human Research Ethics Committee (CAHREC No: HREC-16-406) and the Human Research Ethics Committee (HREC) for the NT Department of Health and Menzies School of Health Research (HREC-16-2599), including an Aboriginal subcommittee. Fully informed verbal consent was obtained from all participants using pictorial information sheets and flipcharts in plain English with Aboriginal language versions available. Verbal consent to participate was used rather than written consent and this was specifically approved by both above ethics committees. Our target population is Indigenous people. Our previous studies suggested that some Indigenous people with low literacy find written forms of communication a disempowering experience. We sought individual verbal consent from each participant after detailed discussion about the purpose, methods and demands, risks and potential benefits of the study (as outlined in the information sheet). We asked the participants if they understood the information provided and if they consented to participation. Their response was recorded in the assessment app by ticking a check box. Participant's willingness to complete the assessment scales and intervention session were further confirmation of their consent to participate.

\section{Consent for publication}

Not applicable.

\section{Competing interests}

KD, TN and DK developed the Stay Strong App which is a paid App. Menzies receives the limited revenue from App sales which is used for maintenance of the App. MS, AC, JH, KH, FB, SB, CS, and SM have no competing interests.

\section{Author details}

'Menzies School of Health Research, Charles Darwin University, Alice Springs, NT 0870, Australia. ${ }^{2}$ Menzies School of Health Research, Charles Darwin University, Darwin, NT 0811, Australia. ${ }^{3}$ Division of Medicine, Royal Darwin Hospital, Darwin, NT 0811, Australia. ${ }^{4}$ Department of Nephrology, Royal Darwin Hospital, Northern Territory Department of Health, Darwin, NT 0810, Australia. ${ }^{5}$ Centre for Children's Health Research and School of Psychology \& Counselling, Faculty of Health, Queensland University of Technology (QUT),
Brisbane, QLD 4101, Australia. ${ }^{6}$ Sydney School of Public Health, Faculty of Medicine and Health, University of Sydney, Sydney, NSW 2006, Australia. ${ }^{7}$ Western Desert Nganampa Walytija Palyantjaku Tjutaku, Alice Springs, NT 0870, Australia. ${ }^{8}$ Central Australian Renal Services, Alice Springs Hospital, Northern Territory Department of Health, Alice Springs, NT 0870, Australia. ${ }^{9}$ Northern Territory Medical Program, Flinders University, Darwin, NT 0815, Australia.

Received: 13 November 2020 Accepted: 5 April 2021

Published online: 19 April 2021

\section{References}

1. Australian Institute of Health and Welfare. Chronic kidney disease [Cat. no. CDK 16]. Canberra: AlHW; 2019.

2. ANZDATA Registry. 42nd report, chapter 10: end stage kidney disease in Aboriginal and Torres Strait islander Australians. Adelaide: Australia and New Zealand Dialysis and Transplant Registry; 2020.

3. Australian Institute of Health and Welfare. Profiles of Aboriginal and Torres Strait Islander people with kidney disease. Cat. no. IHW 229. Canberra: AlHW; 2020.

4. Lawton PD, Cunningham J, Hadlow N, Zhao Y, Jose MD. Chronic kidney disease in the top end of the Northern Territory of Australia, 2002-2011: a retrospective cohort study using existing laboratory data. BMC Nephrol. 2015;16(1):168. https://doi.org/10.1186/s12882-015-0166-6.

5. Devitt J, McMasters A. They don't last long': Aboriginal patient experience of end-stage renal disease in Central Australia. Nephrology. 1998;4(s2):S111-S7. https://doi.org/10.1111/j.1440-1797.1998.tb00485.x.

6. Palmer S, Vecchio M, Craig JC, Tonelli M, Johnson DW, Nicolucci A, et al. Prevalence of depression in chronic kidney disease: systematic review and meta-analysis of observational studies. Kidney Int. 2013;84(1):179-91. https:// doi.org/10.1038/ki.2013.77.

7. Kimmel PL, Peterson RA. Depression in patients with end-stage renal disease treated with dialysis: has the time to treat arrived? Clin J Am Soc Nephrol. 2006;1(3):349-52. https://doi.org/10.2215/CJN.00890306.

8. Patel SS, Peterson RA, Kimmel PL. The impact of social support on endstage renal disease. Semin Dial. 2008;18(2):98-102. https://doi.org/10.1111/j.1 525-139X.2005.18203.x

9. Clelland N, Gould T, Parker E. Searching for evidence: what works in indigenous mental health promotion. Res Evaluation. 2007;18(3):208-16.

10. Firth J, Torous J, Nicholas J, Carney R, Pratap A, Rosenbaum S, et al. The efficacy of smartphone-based mental health interventions for depressive symptoms: a meta-analysis of randomized controlled trials. World Psychiatry. 2017;16(3):287-98. https://doi.org/10.1002/wps.20472.

11. Firth J, Torous J, Nicholas J, Carney R, Rosenbaum S, Sarris J. Can smartphone mental health interventions reduce symptoms of anxiety? A meta-analysis of randomized controlled trials. J Affect Disord. 2017;218:1522. https://doi.org/10.1016/j.jad.2017.04.046.

12. Spijkerman MPJ, Pots WTM, Bohlmeijer ET. Effectiveness of online mindfulness-based interventions in improving mental health: a review and meta-analysis of randomised controlled trials. Clin Psychol Rev. 2016;45:10214. https://doi.org/10.1016/j.cpr.2016.03.009.

13. Dudgeon P, Walker R, Scrine C, Shepherd C, Calma T, Ring I. Effective strategies to strengthen the mental health and wellbeing of Aboriginal and Torres Strait Islander people. Canberra: Australian Institute of Health and Welfare; 2014. Contract No.: Issues paper no 12

14. Nagel T, Robinson G, Condon J, Trauer T. Approach to treatment of mental illness and substance dependence in remote indigenous communities: results of a mixed methods study. Aust J Rural Health. 2009;17(4):174-82. https://doi.org/10.1111/j.1440-1584.2009.01060x.

15. Nagel T, Thompson C. Motivational care planning:self management in indigenous mental health. Aust Fam Physician. 2008;37(12):996-1001.

16. Kowanko I, Kasteel L, Battersby M, Connors C, Nagel T, Trowbridge C, et al. The Flinders AIMhi NT chronic conditions self-management program: evaluation report 2013. Darwin: NT Department of Health; 2013.

17. Dingwall KM, Nagel T, Hughes JT, Kavanagh DJ, Cass A, Howard K, et al. Wellbeing intervention for chronic kidney disease (WICKD): a randomised controlled trial study protocol. BMC Psychol. 2019;7(1):2. https://doi.org/1 0.1186/s40359-018-0264-x.

18. Nagel T, Robinson G, Condon J, Trauer T. An approach to management of psychotic and depressive illness in indigenous communities. Aust J Prim Health Care. 2008;14(1):17-21. https://doi.org/10.1071/PY08003. 
19. Dingwall KM, Puszka S, Sweet M, Nagel T. "Like drawing into sand": acceptability, feasibility and appropriateness of a new e-mental health resource for service providers working with Aboriginal and Torres Strait islander people. Aust Psychol. 2015;50(1):60-9. https://doi.org/10.1111/a p.12100.

20. Nagel T, Sweet M, Dingwall KM, Puszka S, Hughes JT, Kavanagh DJ, et al. Adapting wellbeing research tools for Aboriginal and Torres Strait islander people with chronic kidney disease. BMC Nephrol. 2020;21(1):130. https:// doi.org/10.1186/s12882-020-01776-y.

21. Menzies School of Health Research. Hep B Story Darwin 2014 [Available from: https://itunes.apple.com/au/app/hep-b-story-menzies-school/id9091 78624 ? $\mathrm{mt}=8$.

22. Nagel T, Dingwall KM. AlMhi stay strong planning: brief treatment manual. Darwin: Menzies School Of Health Research; 2014.

23. Andrews G, Slade T. Interpreting scores on the Kessler psychological distress scale (K10). Aust N Z J Public Health. 2001;25(6):494-7. https://doi.org/1 0.1111/j.1467-842X.2001.tb00310.x.

24. Brown A, Mentha R, Rowley KG, Skinner T, Davy C, O'Dea K. Depression in Aboriginal men in central Australia: adaptation of the Patient Health Questionnaire 9. BMC Psychiatry. 2013;13:217 Available from: http://www. biomedcentral.com/1471-244X/13/271.

25. Arean P, Hallgren K, Jordan J, Gazzaley A, Atkins D, Heagerty P, et al. The use and effectiveness of Mobile apps for depression: results from a fully remote clinical trial. J Med Internet Res. 2016;18(12):e330. https://doi.org/1 0.2196/jmir.6482.

26. Hedayati SS, Yalamanchili V, Finkelstein FO. A practical approach to the treatment of depression in patients with chronic kidney disease and endstage renal disease. Kidney Int. 2012;81(3):247-55. https://doi.org/10.1038/ ki.2011.358.

27. Shirazian S, Grant CD, Aina O, Mattana J, Khorassani F, Ricardo AC Depression in chronic kidney disease and end-stage renal disease: similarities and differences in diagnosis, epidemiology, and management. Kidney Int Rep. 2017;2(1):94-107. https://doi.org/10.1016/j.ekir.2016.09.005.

28. Poston JM, Hanson WE. Meta-analysis of psychological assessment as a thereapeutic intervention. Psychol Assess. 2010;22(2):203-12. https://doi. org/10.1037/a0018679.

29. Angell B, Muhunthan J, Eades AM, Cunningham J, Garvey G, Cass A, et al. The health-related quality of life of indigenous populations: a global systematic review. Qual Life Res. 2016;25(9):2161-78. https://doi.org/10.1007/ s11136-016-1311-9.

30. Butler TL, Anderson K, Garvey G, Cunningham J, Ratcliffe J, Tong A, et al. Aboriginal and Torres Strait islander people's domains of wellbeing: a comprehensive literature review. Soc Sci Med. 2019;233:138-57. https://doi. org/10.1016/j.socscimed.2019.06.004.

31. Wuerth D, Finkelstein SH, Finkelstein FO. Psychosocial factors in patients with chronic kidney disease: the identification and treatment of depression in patients maintained on dialysis. Semin Dial. 2005;18(2):142-6. https://doi. org/10.1111/j.1525-139X.2005.18213.X.

32. Campbell M, Fitzpatrick R, Haines A, Kinmonth AL, Sandercock P, Spiegelhalter $\mathrm{D}$, et al. Framework for design and evaluation of complex interventions to improve health. BMJ. 2000;321(7262):694-6. https://doi. org/10.1136/bmj.321.7262.694.

33. Baumeister $H$, Reichler $L$, Munzinger $M$, Lin J. The impact of guidance on internet-based mental health interventions - a systematic review. Internet Interv. 2014; 1(4):205-15. https://doi.org/10.1016/j.invent.2014.08.003.

34. Hobson GR, Caffery L, Neuhaus M, Langbecker DH. Mobile health for first nations populations: systematic review. JMIR Mhealth Uhealth. 2019;7(10): e14877. https://doi.org/10.2196/14877.

35. Jones $\mathrm{L}$, Jacklin $\mathrm{K}$, O'Connell ME. Development and use of health-related Technologies in Indigenous Communities: critical review. J Med Internet Res. 2017;19(7):e256. https://doi.org/10.2196/jmir.7520.

36. Kolewaski CD, Paterson ML, Yeates KE, King-Van Vlack CE. Relocating from the Mushkegowuk territory for hemodialysis: the Cree illness experience and perceived quality of life. Pimatisiwin. 2010;8(3):103-50.

\section{Publisher's Note}

Springer Nature remains neutral with regard to jurisdictional claims in published maps and institutional affiliations.

\section{Ready to submit your research? Choose BMC and benefit from}

- fast, convenient online submission

- thorough peer review by experienced researchers in your field

- rapid publication on acceptance

- support for research data, including large and complex data types

- gold Open Access which fosters wider collaboration and increased citations

- maximum visibility for your research: over $100 \mathrm{M}$ website views per year

At $\mathrm{BMC}$, research is always in progress.

Learn more biomedcentral.com/submissions 